\title{
Handover Measurement in Mobile Cellular Networks: Analysis and Applications to LTE
}

\author{
Van Minh Nguyen*, Chung Shue Chen ${ }^{\dagger}$, Laurent Thomas* \\ *Network and Networking Dept., Alcatel-Lucent Bell Labs, 91620 Nozay, France \\ ${ }^{\dagger}$ TREC, INRIA-ENS, 75214 Paris, France \\ van_minh.nguyen@alcatel-lucent.com, chung-shue.chen@inria.fr, laurent.thomas@alcatel-lucent.com
}

\begin{abstract}
This paper deals with handover measurement in mobile cellular networks. The work is dedicated to network modeling and performance evaluation. The exposition focuses on neighbor cell scanning and addresses its key probabilistic events: (i) suitable handover target found, (ii) service failure, and (iii) scan withdrawal under the interference-limited condition in a multicell system. We derive their expressions and provide a generalized framework for the analysis of handover measurement failure and target cell quality by the best signal quality and minimum duration outage. Results applied to LTE have also shown its effectiveness.
\end{abstract}

\section{INTRODUCTION}

In mobile cellular networks, a user may travel across different cells during a service. Handover (HO) which switches the user's connection from one cell to another is therefore an essential function. Technology advancement is expected to minimize service interrupt and provide seamless handover [1]. A handover procedure includes two important functions which are handover measurement and handover decision-execution [2]. The measurement function is responsible for monitoring the service quality from serving cell and finding a suitable neighbor cell for handover. This consists in measuring some parameters such as cell signal quality or strength. Handover decision-execution is made upon the measurement function. It decides whether a handover is needed and in that case coordinates multi-party handshaking among the user and cells to have handover execution fast and transparent.

Mobile-assisted network-controlled handover is highly recommended in emerging cellular network standards (e.g., LTE [3], LTE-Advanced and WirelessMAN-Advanced [1]) for its operational scalability and effectiveness in which the mobile measures the signal quality of its neighbor cells and reports the measurement result to the network to make handover decision. To indicate the quality of a neighbor cell, the mobile will synchronize with the cell and measure its signal quality which is best represented by the signal-to-interference-plusnoise ratio (SINR).

In this paper, we focus on handover measurement function. The primary goal of handover measurement is to find a suitable handover target before the signal quality from serving cell becomes unsustainable. A call or service will drop if the received SINR falls below a minimum level over a certain period such that the resulting bit error rate is unacceptable. In digital communications, the threshold corresponds to the point at which reliable error correction is no longer possible although a connection may not be torn down [4]. The secondary goal is to at the same time find a high-quality handover target. The neighbor cell with best signal quality among those scanned in each measurement period is in general preferable.

Advanced wireless broadband systems such as $3 \mathrm{G}$ and $4 \mathrm{G}$ [3] allow adjacent cells operating in a common frequency band. The mobile can simultaneously scan several neighbor cells operating in the same frequency, which are thus called intra-frequency cells. The number of cells that the mobile can scan per measurement period is called mobile's measurement capability, say $k$. E.g., a LTE-compliant terminal will be able to scan eight intra-frequency cells in each period $[5, \S 8]$.

It is obvious that higher measurement capability would enhance the performance of the handover measurement and thus that of handover, although this may require more advanced chipset and consume the mobile more resource. Therefore, some basic questions remain to be answered. Most importantly, what is the exact impact of $k$ on the service failure probability and on the quality of target cell? How optimal is the current suggestion (e.g., $k=8$ of LTE standard [5])? Although handover is an important topic and has received a lot of studies, most of them addressed the handover control problem of the handover decision function. The handover measurement function has received less attention and most investigations and analysis are given by simulations. Note that while a handover control problem can be studied conventionally in a simplified model of two cells in which a handover decision is made by assigning the mobile to one of them, the handover measurement problem involves a more complex system in which the signal quality of best cell among a large number of cells needs to be determined. This often incurs modeling and analysis difficulty especially when stochastic parameters are introduced to better describe a wireless network. There lacks a generalized model and analytic study on the performance of the handover measurement which is essential to network design and optimization.

This paper establishes a framework for the system analysis and probabilistic aspect of handover measurement. By observations on stochastic geometry (see, e.g. [6], [7]) and related work on minimum-duration outage [8]-[10], it brings the following main contributions:

1) A generalized model is developed to characterize the handover measurement to facilitate network analysis.

2) The probability of the following key events are derived 
in a multicell system: a suitable handover target found, service failure, and scan withdrawal. Analytical results of the above are known fundamental but challenging.

3) By the above, we investigate the performance of LTE intra-frequency handover measurement in both (i) scanning failure probability and (ii) target cell quality.

\section{System Description}

Poisson point process (p.p.) has received increasing interest [6] and is especially effective in modeling the spatial location of base stations (BSs) seen by a mobile whose location is often unknown to the network operator [11]. The spatial distribution of BSs is modeled by a Poisson p.p. with intensity $\lambda$ in $\mathbb{R}^{2}$.

In the following, we consider that a cell is covered by a BS with omni-directional antenna. The signal strength of BS $i$ received by a mobile at a distance $d_{i}$ is given by:

$$
P_{i}=P_{\mathrm{tx}} d_{i}^{-\beta} Z_{i},
$$

where $P_{\mathrm{tx}}$ is the signal power of common pilot channel for handover measurement and is assumed constant [2], $\beta$ is the path loss exponent, $d_{i}^{-\beta}$ accounts for the far-field path loss, and $Z_{i}=10^{X_{i} / 10}$ denotes lognormal shadowing, where $\left\{X_{i}\right\}$ are assumed independent and identically distributed according to a Gaussian distribution with zero mean and standard deviation $\sigma_{X}<\infty$.

In the time domain, we consider that $X_{i}(t)$ is stationary and has auto-correlation function $R_{X}(\tau)$ with a finite second derivative at the origin (see, e.g., [8]-[10]). Within this context, fast fading is averaged out as it usually varies much faster than that of handover decision process [2]. We will then focus on the above shadowing model. However, relaxing the assumption of lognormal $Z_{i}$ is in general possible to account for different fading scenarios. See, e.g., [7] and [12].

The signal quality of a cell $i$ given by SINR, denoted by $Q_{i}$, is expressible as:

$$
Q_{i}=P_{i} /\left(N_{0}+I_{i}\right)
$$

where $I_{i}=\sum_{j \neq i} P_{j}$, and $N_{0}$ is the thermal noise average power at the receiver and is assumed constant at all $i$.

\section{Handover Measurement}

\section{A. Overall Description}

The details of handover measurement are described below. In principle, the mobile needs a time duration to successfully estimate the signal quality of a neighbor cell. Consequently, the signal quality of neighbor cells are only available at discrete moments. The time spacing between two consecutive moments is called a measurement period, denoted by $T_{\text {meas }}$. E.g., in WCDMA and LTE, the mobile is required to measure eight intra-frequency cells within $T_{\text {meas }}=200 \mathrm{~ms}[5, \S 8]$. By contrast, the signal quality of the serving cell impacts the ongoing service in a time scale as short as one time slot which is usually much smaller than $T_{\text {meas }}$. So, a service failure such as call drop or link outage may occur before the mobile finds a suitable handover target. Therefore, when studying neighbor cell scanning, the signal quality of the serving cell should be

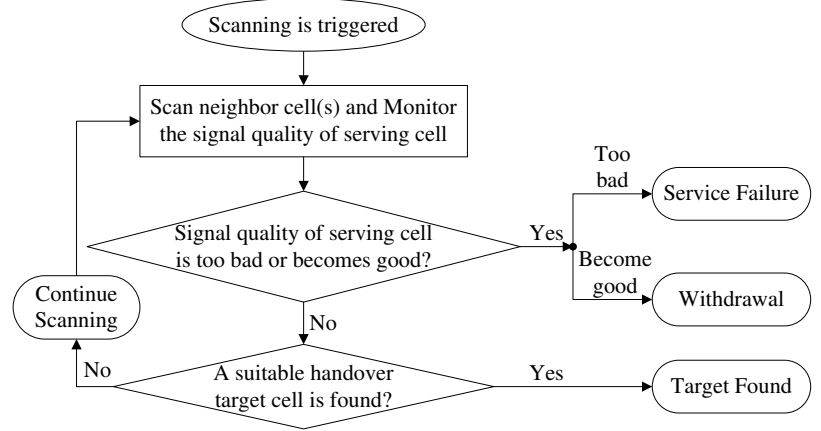

Fig. 1. Handover measurement and neighbor cell scanning

considered in continuous time while that of neighbor cells is determined in the fashion of discrete time instance.

The generic procedure of neighbor cell scanning is shown in Fig. 1. The procedure is initiated as soon as the scanning condition is triggered. The mobile obtains the signal quality of neighbor cells at discrete time $m \times T_{\text {meas }}$, or say moment $m$, where $m=1,2, \ldots$, and $m=1$ corresponds to the first measurement after the scanning is triggered. For notational simplicity, in case of no ambiguity, we will use $m$ and $m T_{\text {meas }}$ interchangeably, and use $[m-1, m]$ to refer to the measurement period $\left[(m-1) T_{\text {meas }}, m T_{\text {meas }}\right]$.

During each $[m-1, m]$, if the signal quality of the serving cell becomes good enough, the mobile will withdraw the scanning. In case the SINR of the serving cell is too bad, the scanning will end in failure as a service interrupt occurs. If the scanning identifies a neighbor cell which has a signal quality better than a certain requirement $\gamma_{\text {req }}$, the cell will be considered as handover target and the cell scanning thus ends in success. Otherwise, the mobile will continue the scanning and keep monitoring the signal quality of the serving BS.

\section{B. Basic Formulation}

1) Suitable Handover Target Found: A neighbor cell is considered as a suitable handover target if its signal quality is better than the required threshold $\gamma_{\text {req. }}$. Note that when one needs to deal with a handover control problem, more criteria may be considered, e.g., whether the signal quality of the target cell is higher than that of the serving cell by a certain margin. Nevertheless, here we deal with the handover measurement function whose role is to find a suitable handover target and prevent service failure. Criteria for handover control problem in handover decision-execution are thus out of the scope here.

As a mobile will scan $k$ cells in each measurement period and the cell with best signal quality is preferable, the event of having a suitable target at moment $m$ can be defined by:

$$
\text { findtarget }_{m}(k) \triangleq\left\{Y_{k} \geq \gamma_{\text {req }}\right\},
$$

where

$$
Y_{k} \triangleq \max _{i=1, \cdots, k} Q_{i}
$$

which denotes the best signal quality received from the $k$ cells. 
2) Service Failure: In wireless communication, the signal usually undergoes time-varying fading and other impairments such as interference. When the SINR stays below a minimum allowable level, say $\gamma_{\min }$, for a long time and successive bits are erroneous, redundancy coding does not help much, leading to a service failure. Therefore, it is more appropriate and also general to incorporate a minimum duration $\tau_{\min }$ when characterizing the event. A service failure during $[m-1, m]$ is thus defined by the following minimum-duration outage:

$$
\begin{aligned}
\text { fail }_{m} \triangleq\{ & Q_{0}(t)<\gamma_{\min }, \forall t \in\left[t_{0}, t_{0}+\tau\right] \text { with } \\
& \left.\tau \geq \tau_{\min } \text { and } t_{0}+\tau_{\min } \in[m-1, m]\right\},
\end{aligned}
$$

where $Q_{0}(t)$ denotes the SINR received from the serving cell at time $t$. Notice that when $\tau_{\min }=0,(5)$ corresponds to an instantaneous SINR outage.

3) Scanning Withdrawal: Handover measurement introduces overheads such as time gaps in data transmission and mobile's resource consumption. It is thus helpful to withdraw scanning when the signal quality of serving cell becomes good enough. However, it is possible that the SINR may rise only for a very short duration and then drops below again. To ensure with high probability that the serving cell's signal quality will be good, the scanning should be canceled only if the serving cell's signal quality is higher than a threshold $\gamma_{\mathrm{w}}$ for a certain period $\tau_{\mathrm{w}}$, e.g. time-to-trigger used in LTE [13]. The event of scanning withdrawal during $[m-1, m]$ is thus defined by:

$$
\begin{array}{r}
\text { wdraw }_{m} \triangleq\left\{Q_{0}(t) \geq \gamma_{\mathrm{w}}, \forall t \in\left[t_{0}, t_{0}+\tau\right]\right. \text { with } \\
\left.\tau \geq \tau_{\mathrm{w}} \text { and } t_{0}+\tau_{\mathrm{w}} \in[m-1, m]\right\} .
\end{array}
$$

\section{Analytical Results of Ho Measurement}

\section{A. Probability of Finding a Suitable Handover Target}

To determine the probability of findtarget ${ }_{m}(k)$, one needs to define the set of candidate cells from which $k$ cells are taken. By today's standards, there are two different cases: limited candidate set and unlimited candidate set. In the former, a mobile only scans neighbor cells belonging to a pre-defined set which includes a certain number of potential candidates, say $N_{\text {cell }}$ cells. E.g., WCDMA and WiMAX networks use a neighbor cell list (NCL) with $N_{\text {cell }}=32$. In the latter, the mobile is allowed to scan any cell in the network. Note that a network may have a very large number of cells, scanning without restriction could introduce unsupportable overhead. Therefore, new broadband cellular systems use a set of, say $N_{\text {CSID }}$, cell synchronization identities (CSID) which allow a mobile to identify and measure cells more easily. When required to scan $k$ cells, the mobile just picks $k$ out of the total $N_{\text {CSID }}$ CSIDs and conduct standardized cell synchronization and measurement. An example of this is LTE that defines 504 physical cell identities (PCI) [3], which serve as CSID. The mobile uses PCIs to scan neighbor cells autonomously [13].

Denote by $B$ the network area of the candidate set. Then $B$ is a bounded area in the case of limited candidate set, while $B=\mathbb{R}^{2}$ in the case of unlimited candidate set. By [7], [14], we determine $\mathbf{P}\left\{\max _{i=1}^{k} Q_{i} \geq \gamma_{\mathrm{req}}\right\}$ for both cases.
1) Case $B=\mathbb{R}^{2}$ : The set of neighbor cells which are scanned through a selection of $k$ out of the totally $N_{\text {CSID }}$ CSIDs can be modeled as a thinning on $B=\mathbb{R}^{2}$, denoted by $S_{k}$, with retention probability $\rho_{k}=k / N_{\text {CSID }}$ [6]. Hence, (4) is equivalent to:

$$
Y_{k} \equiv \max _{i \in S_{k}} Q_{i}
$$

and

$$
\mathbf{P}\left\{\text { findtarget }_{m}(k)\right\}=\bar{F}_{Y_{k}}\left(\gamma_{\text {req }}\right),
$$

where $\bar{F}_{Y_{k}}(\cdot)$ is the tail distribution function of $Y_{k}$. By the result of best signal quality [7, Corollary 5], we can have:

$$
\begin{aligned}
\mathbf{P}\left\{\text { findtarget }_{m}(k)\right\}=\bar{F}_{Y_{k}}\left(\gamma_{\mathrm{req}}\right) & \\
= & \int_{\gamma_{\mathrm{req}}}^{+\infty} \int_{0}^{+\infty} \frac{\exp \left(-C_{1} w^{\alpha}-C_{2}(w, u)\right)}{\pi}\left[-\frac{1+\gamma_{\mathrm{req}}}{\gamma_{\text {req }}}\right. \\
& \times \cos \left(C_{1} w^{\alpha} \tan \left(\frac{\pi \alpha}{2}\right)+C_{3}(w, u)+C_{4}(w, u)\right) \\
& \left.+\cos \left(C_{1} w^{\alpha} \tan \left(\frac{\pi \alpha}{2}\right)+C_{3}(w, u)-w u\right)\right] \mathrm{d} w \mathrm{~d} u,
\end{aligned}
$$

where $\alpha=2 / \beta, C_{1}=c_{\alpha}\left(1-\rho_{k}\right) \Gamma(1-\alpha) \cos \left(\frac{\pi \alpha}{2}\right)$,

$$
\begin{aligned}
& C_{2}(w, u)=\rho_{k} c_{\alpha} u_{1}^{-\alpha}{ }_{1} F_{2}\left(-\frac{\alpha}{2} ; \frac{1}{2}, 1-\frac{\alpha}{2} ;-\frac{u^{2} w^{2}}{4}\right), \\
& C_{3}(w, u)=\rho_{k} c_{\alpha} \frac{\alpha w}{1-\alpha} \frac{{ }_{1} F_{2}\left(\frac{1-\alpha}{2} ; \frac{3}{2}, \frac{3-\alpha}{2} ;-\frac{u^{2} w^{2}}{4}\right)}{u^{\alpha-1}}, \\
& C_{4}(w, u)=w(1-u(1+\gamma) / \gamma),
\end{aligned}
$$

$c_{\alpha}=\pi \lambda\left(P_{\mathrm{tx}} / N_{0}\right)^{\alpha} \exp \left(\alpha^{2} \sigma_{Z}^{2} / 2\right)$ with $\sigma_{Z}=\sigma_{X} \frac{\log 10}{10}, \Gamma(\cdot)$ denotes the gamma function, and ${ }_{1} F_{2}$ denotes the hypergeometric function. Proofs are skipped due to a lack of space. Simulation result in Section V will also show its accuracy.

2) Case $B \subsetneq \mathbb{R}^{2}$ : We consider two different possibilities: scattered networks like rural macro cellular networks where inter-site distance is large such that $\lambda$ is small, and dense networks like urban small cell networks where $\lambda$ is large. Consider $\widehat{B}$ a disk-shaped network area with radius

$$
R_{\widehat{B}}=\sqrt{N_{\text {cell }} /(\pi \lambda)} .
$$

Under the Poisson p.p. assumption of BS spatial distribution, $\widehat{B}$ has on average $N_{\text {cell }}$ BSs. In light of that, we can approximate $B$, the region of the $N_{\text {cell }}$ neighbor cells, by $\widehat{B}$.

For small $\lambda$ and $N_{\text {cell }}$ is relatively large, we can have $R_{\widehat{B}} \approx$ $\infty$, i.e., $\widehat{B}$ can be approximated by $\mathbb{R}^{2}$. Similarly, let $S_{k}$ be a thinning on $\widehat{B}$ with retention probability $\rho_{k}=k / N_{\text {cell }}$ such that $S_{k}$ has on average $k$ cells. The probability of finding a target cell can be well approximated by (9).

For large $\lambda$ (and so the approximation $R_{\widehat{B}} \approx \infty$ is not applicable), denoting the distance from the mobile to its closest neighbor $\mathrm{BS}$ by $R_{\min }>0$, then the $k$ cells scanned are located in a ring-shaped network area with inner and outer radii $R_{\text {min }}$ and $R_{\widehat{B}}$. By [14, Theorem 2], we have:

$$
\begin{aligned}
& \mathbf{P}\left\{\text { findtarget }_{m}(k)\right\}=\bar{F}_{Y_{k}}\left(\gamma_{\text {req }}\right) \\
& \approx \int_{\gamma_{\text {req }}}^{\infty}\left\{f_{M_{k}}(u) \int_{0}^{\infty} \frac{2}{\pi w} e^{-\delta w^{\alpha}} \sin \left(w \frac{u-\gamma_{\text {req }}}{2 \gamma_{\text {req }}}\right)\right. \\
& \left.\quad \times \cos \left(w u+w \frac{u-\gamma_{\text {req }}}{2 \gamma_{\text {req }}}-\delta w^{\alpha} \tan \frac{\pi \alpha}{2}\right) \mathrm{d} w\right\} \mathrm{d} u,
\end{aligned}
$$


where $\delta=c_{\alpha} \Gamma(1-\alpha) \cos (\pi \alpha / 2)$, and $f_{M_{k}}$ is the probability density function (pdf) of random variable $M_{k}=\max _{i=1}^{k} P_{i}$ :

$$
f_{M_{k}}(x)=k \cdot f_{P}(x) \cdot F_{P}^{k-1}(x),
$$

with $F_{P}$ denoting the cumulative density function (cdf) of the signal strength received from a cell in $\widehat{B}$ is given by

$$
F_{P}(x)=c\left(\frac{G_{1}(x)}{a^{\alpha}}-\frac{G_{2}(x)}{b^{\alpha}}-\frac{e^{\omega} G_{3}(x)}{x^{\alpha}}+\frac{e^{\omega} G_{4}(x)}{x^{\alpha}}\right),
$$

where $a=\left(P_{\mathrm{tx}} / N_{0}\right) R_{\widehat{B}}^{-\beta}, \quad b=\left(P_{\mathrm{tx}} / N_{0}\right) R_{\min }^{-\beta}, c=$ $\left(P_{\mathrm{tx}} / N_{0}\right)^{\alpha} /\left(R_{\widehat{B}}^{2}-R_{\min }^{2}\right), \omega=2 \sigma_{X}^{2} / \beta^{2}$, and $G_{j=1,2,3,4}$ refers to the cdf of lognormal distribution of parameters $\left(\mu_{j}, \sigma_{X}\right)$, where $\mu_{1}=\log a, \mu_{2}=\log b, \mu_{3}=\mu_{1}+\alpha \sigma_{X}^{2}$, and $\mu_{4}=\mu_{2}+\alpha \sigma_{X}^{2} \cdot f_{P}(x)=\mathrm{d} F_{P}(x) / \mathrm{d} x$. It is observed that (11) is a good approximation [14].

\section{B. Probability of Service Failure}

Recall that $X(t)$ is a Gaussian process for shadowing. By substituting (1) and (2), the event in (5) can be re-written as:

$$
Q_{0}(t)<\gamma_{\min } \Leftrightarrow X(t)<\hat{\gamma}_{\min }(t),
$$

where

$$
\hat{\gamma}_{\min }(t)=10 \log _{10}\left(\gamma_{\min }\left(N_{0}+I(t)\right) d(t)^{\beta} / P_{\mathrm{tx}}\right) .
$$

By (13), the service failure probability $\mathbf{P}\left\{f a i l_{m}\right\}$ is thus given by the minimum-duration outage of $X(t)$ w.r.t. $\hat{\gamma}_{\min }(t)$ and $\tau_{\text {min }}$, considering $\left[(m-1) T_{\text {meas }}-\tau_{\text {min }}, m T_{\text {meas }}\right]$. Note that $\tau_{\text {min }}$ and $T_{\text {meas }}$ are typically about a few hundreds of milliseconds [5]. The above interval, i.e., $T_{\text {meas }}+\tau_{\text {min }}$, is very short so that we consider that in which the distance between the mobile and its serving BS is constant, denoted $d_{m}$.

Following [8]-[10], given that $R_{X}(\tau)$ has a finite second derivative at the origin, $d(t)=d_{m}$, and if the interference is constant, say $x_{0}$, we can have the following closed-form expression of the outage probability by the asymptotic properties of the level-crossing of a stationary Gaussian process:

$$
\begin{aligned}
& \mathbf{P}\left\{\text { fail }_{m} \mid I(t)=x_{0}, d(t)=d_{m}\right\}=\mathbf{P}\left\{\text { outage w.r.t. } \hat{\gamma}_{\min }\right\} \\
& \quad=\eta_{\min }\left[\tau_{\min } \exp \left(-\frac{\tau_{\min }^{2}}{2 \sigma_{\min }^{2}}\right)+\sqrt{2 \pi} \sigma_{\min } q\left(\frac{\tau_{\min }}{\sigma_{\min }}\right)\right],
\end{aligned}
$$

where $q(x)=\int_{x}^{\infty} \frac{\exp \left(-\frac{t^{2}}{2}\right)}{\sqrt{2 \pi}} \mathrm{d} t, \sigma_{\min }$ is the parameter of the Rayleigh distribution describing the fade duration and is given by:

$$
\sigma_{\min }=\sqrt{2 / \pi} q\left(-\hat{\gamma}_{\min } / \sigma_{X}\right) / \eta_{\min },
$$

and $\eta_{\min }$ is the down-level crossing rate given by:

$$
\eta_{\min }=\sqrt{\nu} /\left(2 \pi \sigma_{X}\right) \exp \left(-\hat{\gamma}_{\min }^{2} /\left(2 \sigma_{X}^{2}\right)\right),
$$

where $\nu=-\left.\frac{\mathrm{d}^{2}}{\mathrm{~d} \tau^{2}} R_{X}(\tau)\right|_{\tau=0}$.

Note that in practice, the interference behaves as a random variable. By the stochastic model described in Section II, the interference field $I(t)$ can be modeled as a shot noise on $\mathbb{R}^{2}$ [6] and has the following characteristic function [7], [11]:

$$
\phi_{I}(w)=\exp \left(-\delta|w|^{\alpha}[1-j \operatorname{sign}(w) \tan (\pi \alpha / 2)]\right),
$$

where $\delta$ is defined under (11). Consider that $\beta>2$, we have $0<\alpha<1$ such that $\phi_{I}(w)$ is absolutely integrable. By [15, Thm.3, p.509], the pdf of $I$ is thus given by:

$$
f_{I}(x)=\frac{1}{\pi} \int_{0}^{\infty} e^{-\delta w^{\alpha}} \cos \left(\delta \tan \left(\frac{\pi \alpha}{2}\right) w^{\alpha}-x w\right) \mathrm{d} w .
$$

A more general expression of the service failure probability with time-varying random interference $I(t)$ is expressible as:

$$
\begin{gathered}
\mathbf{P}\left\{\text { fail }_{m} \mid d=d_{m}\right\}=\int_{0}^{\infty} \mathbf{P}\left\{\text { fail }_{m} \mid I=x, d=d_{m}\right\} f_{I}(x) \mathrm{d} x \\
=\int_{0}^{\infty} \int_{0}^{\infty} \frac{\eta_{\min }}{\pi}\left(\tau_{\min } e^{-\frac{\tau_{\min }^{2}}{2 \sigma_{\min }^{2}}}+\sqrt{2 \pi} \sigma_{\min } q\left(\frac{\tau_{\min }}{\sigma_{\min }}\right)\right) \\
\times e^{-\delta w^{\alpha}} \cos \left(\delta \tan \left(\frac{\pi \alpha}{2}\right) w^{\alpha}-x w\right) \mathrm{d} w \mathrm{~d} x .
\end{gathered}
$$

\section{Probability of Scanning Withdrawal}

Similarly to (13) and (14), (6) can be re-written as:

$$
Q_{0}(t) \geq \gamma_{\mathrm{w}} \Leftrightarrow-X(t) \leq \hat{\gamma}_{\mathrm{w}}(t)
$$

where $-X(t)$ is still a stationary Gaussian process, and

$$
\hat{\gamma}_{\mathrm{w}}(t)=-10 \log _{10}\left(\gamma_{\mathrm{w}}\left(N_{0}+I(t)\right) d(t)^{\beta} / P_{\mathrm{tx}}\right) .
$$

Consequently, the scanning withdrawal event is given by the minimum-duration outage of $-X(t)$ w.r.t. $\hat{\gamma}_{\mathrm{w}}(t)$ and $\tau_{\mathrm{w}}$. Since the interval $T_{\text {meas }}+\tau_{\mathrm{w}}$ is also very short, we consider that during $\left[(m-1) T_{\text {meas }}-\tau_{\mathrm{w}}, m T_{\text {meas }}\right]$, the distance between the mobile and its serving BS is constant. Provided that $R_{X}$ has a finite second derivative at the origin and $I(t)=x_{0}$,

$$
\begin{gathered}
\mathbf{P}\left\{\text { wdraw }_{m} \mid I(t)=x_{0}, d(t)=d_{m}\right\}=\mathbf{P}\left\{\text { outage w.r.t. } \hat{\gamma}_{\mathrm{w}}\right\} \\
=\eta_{\mathrm{w}}\left[\tau_{\mathrm{w}} \exp \left(-\frac{\tau_{\mathrm{w}}^{2}}{2 \sigma_{\mathrm{w}}^{2}}\right)+\sqrt{2 \pi} \sigma_{\mathrm{w}} q\left(\frac{\tau_{\mathrm{w}}}{\sigma_{\mathrm{w}}}\right)\right]
\end{gathered}
$$

where $\eta_{\mathrm{w}}=\frac{\sqrt{\nu}}{2 \pi \sigma_{X}} e^{-\hat{\gamma}_{\mathrm{w}}^{2} /\left(2 \sigma_{X}^{2}\right)}$ and $\sigma_{\mathrm{w}}=\sqrt{\frac{2}{\pi}} \frac{q\left(-\hat{\gamma}_{\mathrm{w}} / \sigma_{X}\right)}{\eta_{\mathrm{w}}}$. Similarly to (20), by (19) we obtain a more general expression:

$$
\begin{aligned}
& \mathbf{P}\left\{\text { wraw }_{m} \mid d(t)=d_{m}\right\} \\
& =\int_{0}^{\infty} \int_{0}^{\infty} \frac{\eta_{\mathrm{w}}}{\pi}\left(\tau_{\mathrm{w}} e^{-\frac{\tau_{\mathrm{w}}^{2}}{2 \sigma_{\mathrm{w}}^{2}}}+\sqrt{2 \pi} \sigma_{\mathrm{w}} q\left(\frac{\tau_{\mathrm{w}}}{\sigma_{\mathrm{w}}}\right)\right) \\
& \quad \times e^{-\delta w^{\alpha}} \cos \left(\delta \tan \left(\frac{\pi \alpha}{2}\right) w^{\alpha}-x w\right) \mathrm{d} w \mathrm{~d} x .
\end{aligned}
$$

\section{APPLICATION to LTE INTRA-FREQUENCY HO}

In the following, we study the performance of LTE intrafrequency handover measurement by the above results. A mobile in LTE continuously scans intra-frequency cells during its connected mode. This corresponds to the case $\gamma_{\mathrm{w}}=$ $+\infty$ and so $\mathbf{P}\left\{\right.$ wdraw $\left._{m}\right\}=0$. The mobile scans neighbor cells autonomously using 504 PCIs without a NCL [3], [5]. $\mathbf{P}\left\{\right.$ findtarget $\left._{m}(k)\right\}$ is thus given by (9) with retention probability $\rho_{k}=k / 504$.

Fig. 2 describes the temporal evolution of the handover measurement. It is composed of Fail, Scan and TargetFound, which correspond to the states of service failure, scanning continuation, and a suitable handover target found, respectively. Note that when being in Fail and TargetFound, the current 


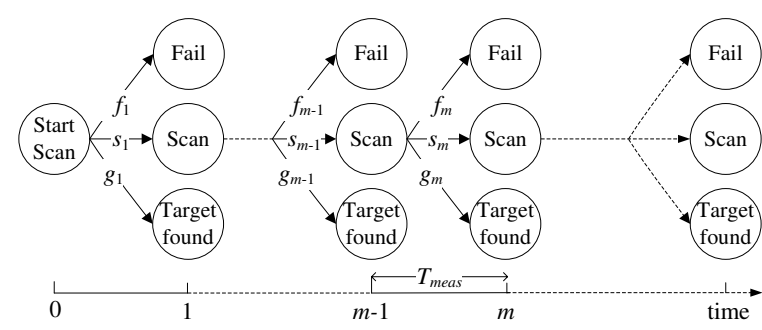

Fig. 2. Temporal evolution of the handover measurement

scan terminates since the mobile will either (i) in Fail, perform a new process of link re-establishment, or (ii) in TargetFound, conduct handover decision-execution. It is clear that they are the absorbing states.

The transition probabilities from Scan at $m-1$ to states

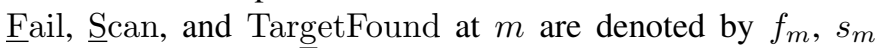
and $g_{m}$, respectively. It is clear that they are given by:

$$
\begin{aligned}
& f_{m}=\mathbf{P}\left\{\text { fail }_{m}\right\}, \\
& g_{m}=\left(1-f_{m}\right) \times \mathbf{P}\left\{\text { findtarget }_{m}(k)\right\}, \\
& \left.s_{m}=\left(1-f_{m}\right) \times\left(1-\mathbf{P} \text { findtarget }_{m}(k)\right\}\right) .
\end{aligned}
$$

\section{A. Performance Metric}

We investigate the system performance. As shown in Fig. 2, an occurrence of service failure at $m$ is given by $\left\{\operatorname{Scan}_{1}, \ldots, \operatorname{Scan}_{m-1}\right.$, Fail $\left._{m}\right\}$. Thus,

$\mathbf{P}\{$ scanning fails at $m\}=\left(s_{1} \times \cdots \times s_{m-1}\right) \times f_{m}$.

The system's scanning effectiveness can be indicated by its asymptotic scanning failure probability expressible as:

$$
\mathcal{F}(k)=\lim _{M \rightarrow \infty} \sum_{m=1}^{M}\left(f_{m} \prod_{l=1}^{m-1} s_{l}\right),
$$

where $M$ represents the time duration of the on-going service.

Note that $\mathcal{F}(k)$ characterizes the possibility that the scanning procedure cannot identify a suitable target cell before a service failure occurs. However, finding target cell with high quality is as well desirable. Therefore, it is necessary to assess the target cell quality when the scanning is in success.

As a suitable target cell is given by the best cell among $k$ cells scanned and provided that its signal quality is better than $\gamma_{\text {req }}$, the resulting signal quality is thus given by $\mathbf{E}\left\{Y_{k} \mid Y_{k} \geq\right.$ $\left.\gamma_{\text {req }}\right\}$. Therefore, the tail distribution $\bar{F}_{Y_{k}}(y)$ is given by

$$
\bar{F}_{Y_{k} \mid Y_{k} \geq \gamma_{\text {req }}}(y)=\mathbf{1}\left(y<\gamma_{\text {req }}\right)+\mathbf{1}\left(y \geq \gamma_{\text {req }}\right) \frac{\bar{F}_{Y_{k}}(y)}{\bar{F}_{Y_{k}}\left(\gamma_{\text {req }}\right)},
$$

where $\mathbf{1}(\cdot)$ is the indicator function. Hence,

$$
\mathbf{E}\left\{Y_{k} \mid Y_{k} \geq \gamma_{\text {req }}\right\}=\gamma_{\text {req }}+\int_{\gamma_{\text {req }}}^{\infty} \frac{\bar{F}_{Y_{k}}(y) \mathrm{d} y}{\bar{F}_{Y_{k}}\left(\gamma_{\text {req }}\right)} .
$$

The scanning is in success when a suitable target cell is found, i.e., $\left\{\operatorname{Scan}_{1}, \ldots, \operatorname{Scan}_{m-1}, \operatorname{TargetFound}_{m}\right\}$. Thus,

$$
\mathbf{P}\{\text { Target found at } m\}=\left(s_{1} \times \cdots \times s_{m-1}\right) \times g_{m} .
$$

TABLE I

SYSTEM PARAMETERS

\begin{tabular}{c|c|c|c}
\hline Parameter & Assumption & Parameter & Assumption \\
\hline \hline BS's tx. power, $P_{\mathrm{BS}}$ & $43 \mathrm{dBm}$ & Cell radius, $R$ & $700 \mathrm{~m}$ \\
Std. deviation, $\sigma_{X}$ & $10 \mathrm{~dB}$ & Noise density & $-174 \mathrm{dBm} / \mathrm{Hz}$ \\
Corr. distance, $d_{c}$ & $50 \mathrm{~m}$ & Bandwidth, $W$ & $20 \mathrm{MHz}$ \\
User's velocity, $v$ & $10 \mathrm{~m} / \mathrm{s}$ & UE noise figure, $N_{\mathrm{F}}$ & $9 \mathrm{~dB}$ \\
Min duration, $\tau_{\text {min }}$ & $200 \mathrm{~ms}$ & Min level, $\gamma_{\text {min }}$ & -20 to $-5 \mathrm{~dB}$ \\
Meas. period, $T_{\text {meas }}$ & $200 \mathrm{~ms}$ & HO margin, $\Delta_{\mathrm{HO}}$ & $2 \mathrm{~dB}$ \\
Path loss & \multicolumn{2}{|c}{$L(d)=15.3+37.6 \log _{10} d,(d$ is in meters $)$} \\
\hline
\end{tabular}

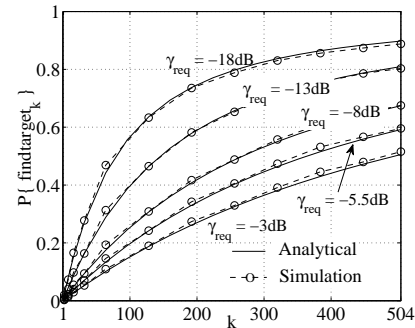

(a) $\mathbf{P}\left\{\right.$ findtarget $\left._{k}\right\}$

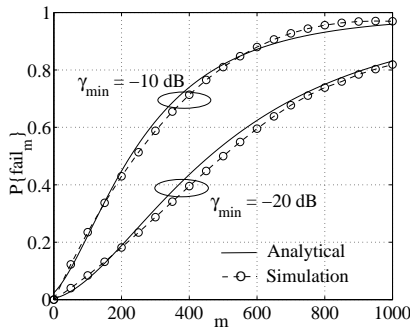

(b) $\mathbf{P}\left\{\right.$ fail $\left._{m}\right\}$
Fig. 3. Validation: analytical expression against simulation result

It follows that the quality of the scanning is measurable by the asymptotic target cell quality defined below:

$$
\mathcal{Q}(k)=\lim _{M \rightarrow \infty} \sum_{m=1}^{M} \mathbf{P}\{\text { Target found at } m\} \mathbf{E}\left\{Y_{k} \mid Y_{k} \geq \gamma_{\text {req }}\right\},
$$

which, by (31) and (32), is expressible as:

$$
\mathcal{Q}(k)=\left(\lim _{M \rightarrow \infty} \sum_{m=1}^{M} g_{m} \prod_{l=1}^{m-1} s_{l}\right)\left(\gamma_{\text {req }}+\int_{\gamma_{\text {req }}}^{\infty} \frac{\bar{F}_{Y_{k}}(y) \mathrm{d} y}{\bar{F}_{Y_{k}}\left(\gamma_{\mathrm{req}}\right)}\right) .
$$

\section{B. Simulation Setting}

System parameters are summarized in Table I following 3GPP recommendations [5], [16] for LTE urban macro cellular networks. The network density $\lambda$ is set corresponding to hexagonal cellular layout such that $\lambda=2 /\left(3 \sqrt{3} R^{2}\right) \mathrm{BS} / \mathrm{m}^{2}$. In lognormal shadowing [8]-[10],

$$
R_{X}(\tau)=\sigma_{X}^{2} \exp \left(-(1 / 2)\left(v \tau / d_{c}\right)^{2}\right),
$$

where $v$ is the user's velocity. So, $\nu$ under (17) is given by:

$$
\nu=-\left.R_{X}^{\prime \prime}(\tau)\right|_{(\tau=0)}=\sigma_{X}^{2}\left(v / d_{c}\right)^{2} .
$$

We study relative and absolute requirement policies; both are considered in LTE [13]. In the former, $\gamma_{\text {req }}$ is required to be higher than $\gamma_{\min }$ by a $\mathrm{HO}$ margin $\Delta_{\mathrm{HO}}$ such that $\gamma_{\text {req }}=$ $\gamma_{\text {min }}+\Delta_{\text {HO }}$. In the latter, $\gamma_{\text {req }}$ is on a fixed absolute level.

Regarding user mobility, we considered 100 mobile users in the serving cell and each of them moves away from the serving BS at velocity $v$ in a random direction generated according to a uniform distribution on $(-\pi, \pi]$. This scenario has been considered as the most critical circumstance [16]. 


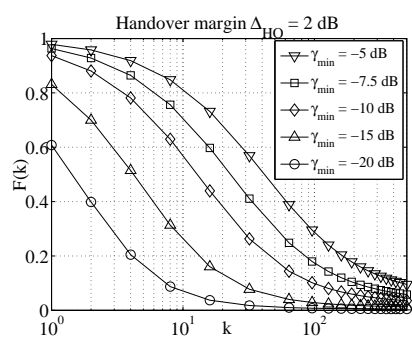

(a) Under relative requirement

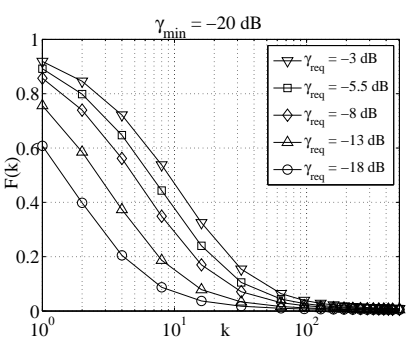

(b) Under absolute requirement
Fig. 4. Asymptotic scanning failure probability $\mathcal{F}(k)$

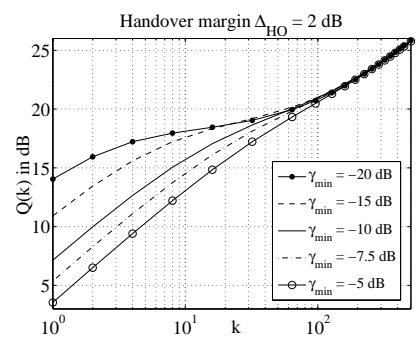

(a) Under relative requirement

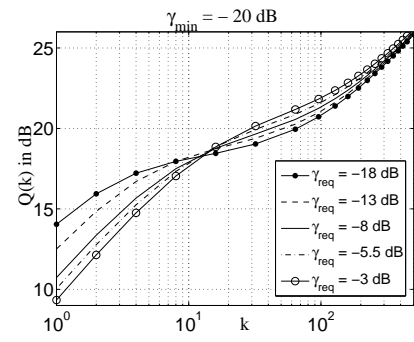

(b) Under absolute requirement
Fig. 5. Asymptotic target cell quality $\mathcal{Q}(k)$

\section{Numerical Result}

First, Fig. 3 verifies our analytical model against computer simulation. Fig. 3(a) plots $\mathbf{P}\left\{\right.$ findtarget $\left._{k}\right\}$ of (9), which corresponds to common LTE model. Its agreement with simulation illustrates the accuracy of modeling the best signal quality $Y_{k}$ defined in (4) by the maximum of SINRs received from the thinning $S_{k}$ proposed in (7). Fig. 3(b) checks the derived probability of minimum-duration outage $\mathbf{P}\left\{\right.$ fail $\left._{m}\right\}$. Results show that the analytical model is close to the simulation in both settings. The lack of space did not allow to show here comparisons of the other probabilities. However, we observed that analytical and simulation results are well matched.

Fig. 4 illustrates that high measurement capability reduces the scanning failure probability $\mathcal{F}(k)$. It also indicates $\mathcal{F}(k)$ 's dependence on $\gamma_{\min }$ and $\gamma_{\text {req }}$ respectively. Comparing Fig. 4(b) and 4(a), absolute requirement policy shows better robustness than relative requirement policy to different system configurations. Note that the former uses a fixed and generally lower $\gamma_{\text {min }}$. Fig. 4(b) shows that increasing $k$ to the order of $10^{2}$ improves the performance very marginally. Besides, we can see that the current LTE requirement of $k=8$ is insufficient for reliable handover performance (here, no pre-defined NCL).

Fig. 5 shows the resulting target cell quality. In Fig. 5(a), lower $\gamma_{\text {min }}$ (and so lower $\gamma_{\text {req }}$ ) always results in higher target cell quality $\mathcal{Q}(k)$ under the relative requirement scheme. On the other hand, Fig. 5(b) shows a crossing point between the curves when $k$ is in-between 10 and 16 under the absolute requirement scheme, in which given large $k, \mathcal{Q}(k)$ can be generally enhanced by higher $\gamma_{\text {req }}$. However, for small $k$, it is better to maintain low $\gamma_{\text {req }}$. Fig. 5 allows the network operator to set $\gamma_{\text {req }}$ parameter so as to obtain the best performance.

\section{CONCLUding REMARKS}

We focus on the analytical modeling of handover measurement function in emerging broadband 3G/4G networks with fundamental establishment in the probabilistic aspects of best signal quality and minimum-duration outage. We derive the probability of the key events in a multicell system: (i) suitable handover target found, (ii) service failure, and (iii) scan withdrawal. Particularly, (ii) and (iii) are derived by minimum-duration outage under interference-limited condition which is in fact more general and realistic and can cover the commonly used instantaneous SINR outage as special case. A generalized framework of HO measurement is provided for network design and optimization purposes. Results indicated that the LTE's current requirement for UE intra-frequency measurement capability with $k=8$ seems insufficient for reliable $\mathrm{HO}$ measurement without using a neighbor cell list.

\section{ACKNOWLEDGMENT}

This work was done within the framework of the joint laboratory of Alcatel-Lucent Bell Labs and INRIA. Authors would like to thank François Baccelli for his valuable discussions and continuous supports in this work.

\section{REFERENCES}

[1] R. Y. Kim, I. Jung, X. Yang, and C.-C. Chou, "Advanced handover schemes in IMT-advanced systems [WiMAX/LTE Update]," IEEE Communications Magazine, vol. 48, no. 8, pp. 78-85, Aug. 2010.

[2] M. Anas, F. Calabrese, P.-E. Ostling, K. Pedersen, and P. Mogensen, "Performance analysis of handover measurements and layer 3 filtering for UTRAN LTE," in IEEE PIMRC, Sept. 2007, pp. 1-5.

[3] 3GPP TS 36.300, "Evolved Universal Terrestrial Radio Access (EUTRA) and Evolved Universal Terrestrial Radio Access Network (EUTRAN) - Overall description: Stage 2," Tech. Spec. v9.4.0, Jun 2010

[4] V. Veeravalli and O. Kelly, "A locally optimal handoff algorithm for cellular communications," IEEE Trans. Veh. Technol., vol. 46, no. 3, pp. 603-609, Aug 1997.

[5] 3GPP TS 36.133, "Evolved Universal Terrestrial Radio Access: Requirements for support of Radio Resource Management," Tech. Spec. v9.4.0.

[6] F. Baccelli and B. Blaszczyszyn, Stochastic Geometry and Wireless Networks, Volume I - Theory. NoW Publishers, 2009.

[7] V. M. Nguyen and F. Baccelli, "A stochastic geometry model for the best signal quality in a wireless network," in 6th International Workshop on Spatial Stochastic Models for Wireless Networks, Jun. 2010.

[8] N. B. Mandayam, P.-C. Chen, and J. M. Holtzman, "Minimum duration outage for CDMA cellular systems: A level crossing analysis," Wireless Personal Commun., Springer, vol. 7, no. 2-3, pp. 135-146, Aug. 1998.

[9] F. Graziosi and F. Santucci, "Distribution of outage intervals in macrodiversity cellular systems," IEEE JSAC, vol. 17, no. 11, 1999.

[10] H. Jiang and C. Davis, "Cell-coverage estimation based on duration outage criterion for CDMA cellular systems," IEEE Trans. Veh. Technol., vol. 52, no. 4, pp. $814-822$, Jul. 2003.

[11] M. Z. Win, P. C. Pinto, and L. A. Shepp, "A mathematical theory of network interference and its applications," Proceedings of the IEEE, vol. 97, no. 2, pp. 205-230, Feb. 2009.

[12] J. Lai and N. Mandayam, "Minimum duration outages in Rayleigh fading channels," IEEE Trans. Commun., vol. 49, no. 10, pp. 1755-1761, 2001.

[13] 3GPP TS 36.331, "Evolved Universal Terrestrial Radio Access Radio Resource Control: Protocol specification,” Tech. Spec. v9.3.0, Jun 2010.

[14] V. M. Nguyen, F. Baccelli, L. Thomas, and C. S. Chen, "Best signal quality in cellular networks: asymptotic properties and applications to mobility management in small cell networks," EURASIP Journal on Wireless Communications and Networking, pp. 1-14, Jun. 2010.

[15] W. Feller, An Introduction to Probability Theory and its Applications. John Wiley \& Sons, 1971, vol. 2.

[16] 3GPP TR 36.942, "Evolved Universal Terrestrial Radio Access: Radio Frequency System Scenarios,” Tech. Rep. v8.2.0, Jun 2010. 